\title{
Erosion des sédiments et vague générées par le passage des bateaux : mesures et analyses
}

\author{
Damien PHAM VAN BANG ${ }^{1,2}$, Abdellatif OUAHSINE ${ }^{3}$, \\ Philippe SERGENT ${ }^{2}$, Bernard LONG ${ }^{4}$, Stéphane MONTREUIL ${ }^{4}$, \\ Pierre DEBAILLON ${ }^{2}$, François HISSEL ${ }^{2}$
}

${ }^{1}$ : Laboratoire d'Hydraulique Saint Venant (Univ. Paris-Est, Laboratoire commun EDF/R\&D-CETMEF-Ecole des Ponts), 6 quai Watier, Bat. K., BP 49,

F-78401 Chatou Cedex, damien.pham-van.bang@edf.fr

2 : Centre d'Etudes Techniques Maritimes et Fluviales (CETMEF),

2 bd Gambetta, BP 60039, F-60321 Compiègne Cedex, philippe.sergent@developpement-durable.gouv.fr

3 : Laboratoire commun d'Hydraulique Numérique (UTC-CETMEF-CNRS UMR 6253), Centre de Recherche Le Royallieu, BP 20529, F-60205 Compiègne abdellatif.ouahsine@utc.fr

4 : Institut National de Recherche Scientifique, Eau-Terre-Environnement (INRS-ETE), Université du Québec, 490 de la Couronne, Québec G1K 9A9, Canada, blong@ete-inrs.ca

\section{Résumé :}

Une campagne in situ pour mesurer l'impact du passage des bateaux sur l'entretien des voies navigables est présentée. Le fond et les berges d'un canal artificiel et d'une rivière naviguée sont instrumentés par des courantomètres, des turbidimètres et des capteurs de hauteurs d'eau. La re-suspension des sédiments au passage des bateaux a été mesurée sur le fond et à l'arrière des bateaux. Les vagues générées par les bateaux sont obtenues à proximité des berges par un dispositif à trois points.

\begin{abstract}
:
A field experiment to measure the impact of passing boats on the maintenance of waterways is presented. The bottom and the banks of an artificial channel and a navigated river are instrumented by currentmeters, turbidimeters and water level sensors. Sediment resuspension induced by passing boats are measured at the bottom and behind the boats. The waves generated by boats are obtained close to the banks by a three point device.
\end{abstract}

Mots-clés : Batillage - remise en suspension - érosion des berge. 


\section{$1 \quad$ Introduction}

Le transport maritime et fluvial est très attractif puisqu'il répond pleinement aux objectifs de développement durable. En effet, ce mode de transport permet de réduire la saturation des routes et des autoroutes et de diminuer les émissions de $\mathrm{CO}_{2}$; il offre un coût à la tonne transportée très compétitif ainsi que des loisirs aux riverains et aux touristes (promenades pour piétons/cyclistes).

Toutefois, la navigation a des impacts, plus ou moins directs, sur le milieu aquatique. Elle induit des vitesses de courants susceptibles de nuire à certains poissons (WOLTHER \& ARLINGHAUS, 2003). La remise en suspension des sédiments (GARCIA et al., 1999) génère des turbidités empêchant potentiellement la photosynthèse ou risquant d'ensevelir les habitats aquatiques. Enfin, l'érosion des berges génère des coûts importants pour l'entretien de la voie d'eau (KOSLOWSKI, 2007).

La liaison fluviale à grands gabarits 'Seine-Nord Europe', inscrite parmi les projets prioritaires européens, aura pour conséquence une modification dans la nature et dans la fréquence du trafic. Afin de préconiser des recommandations sur la navigation (MOULIN et al., 2003) ou sur la technique pour protéger les berges (ROCHETTE, 2008), une campagne expérimentale est nécessaire afin de quantifier la re-suspension des sédiments et le batillage.

Dans un premier temps, nous présentons les sites d'étude, la méthodologie et les instruments déployés. Puis les résultats portant sur les remises en suspension sédimentaires sont présentés. Enfin, les mesures de batillages sont illustrés avec des analyses sur la direction des ondes, leur fréquence et leur amplitude.

\section{$2 \quad$ Présentation de la campagne expérimentale}

\subsection{Objectifs de l'étude}

La campagne de relevés de terrain vise à caractériser l'impact du passage des bateaux sur le fond et les berges des voies navigables. D'après le groupe de travail $\mathrm{n}^{\circ} 27$ de l'Association Internationale de la Navigation (AIPCN), ces impacts dépendent du type, de la direction et de la vitesse des bateaux mais aussi de la nature de la voie d'eau (type du fond et de berges).

Les principaux objectifs de la campagne, réalisée sur deux semaines de novembre 2006, consistent à mesurer lors des passages des bateaux : (i) l'érosion des fonds ; (ii) le panache sédimentaire et (iii) le batillage.

D'une part, des capteurs posés sur le fond de la voie navigable permettent d'obtenir des informations sur le courant et la concentration des sédiments pour caractériser la remise en suspension. D'autre part, des mesures de concentration sur la colonne d'eau sont réalisées à l'arrière et à différentes distances des bateaux. En outre, des jauges de hauteurs d'eau sont disposées à proximité des 
berges. Enfin, les informations sur le trafic (type de bateaux, caractère chargé ou non, vitesse et direction) sont notées pour relier les causes aux effets.

\subsection{Les deux sites 'Seine-Nord'}

Dans le contexte du projet 'Seine-Nord-Europe', deux sites (Fig. 1) ont été retenus. Le premier est situé sur une ligne droite $(50 \mathrm{~m}$ de large, $4 \mathrm{~m}$ de profondeur) du canal de la Sensée (PK 10, bief de partage) : les berges sont constituées de parois verticales en maçonnerie. En l'absence de bateau, le courant est quasi-nul. Le second est situé sur un bras de Seine en zone NATURA 2000 ( $150 \mathrm{~m}$ de large, entre 6 et $13 \mathrm{~m}$ de profondeur) avec des berges naturelles et un courant non nul.

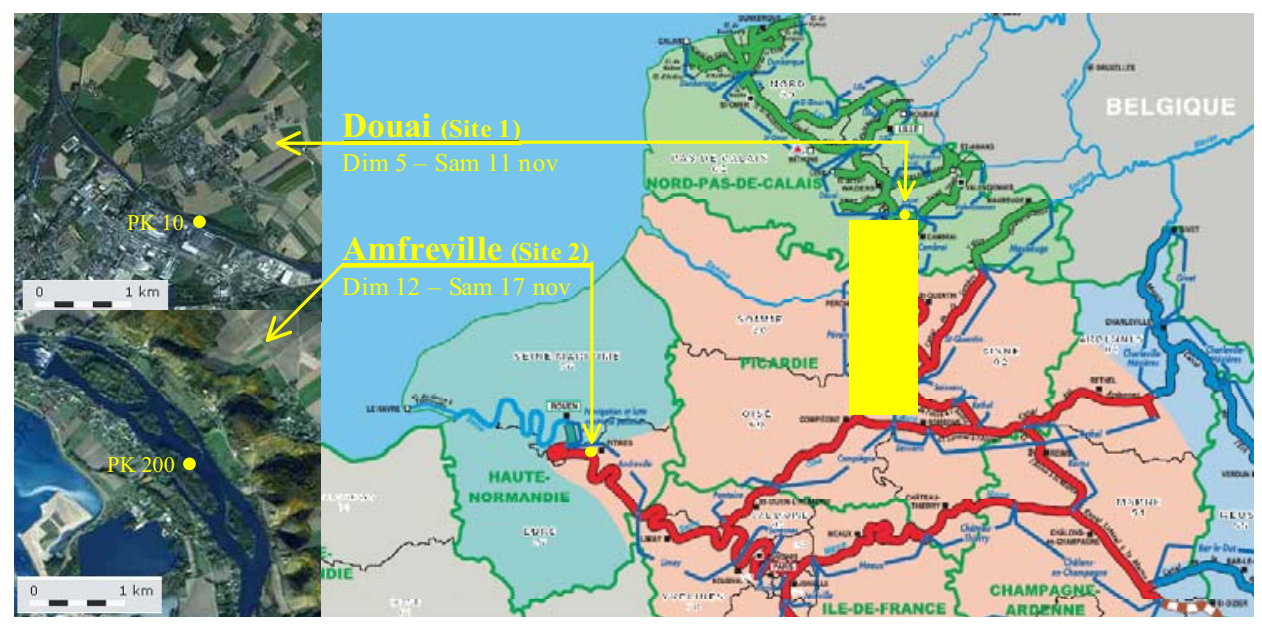

Figure 1 : Localisation des deux sites 'Seine-Nord'retenus pour l'étude.

\subsection{Protocole expérimental}

Les figures 2 présentent la procédure (méthodologie et métrologie) de mesure qui s'articule autour de trois systèmes aux fonctionnalités suivantes :

- vedette de travail pour mesurer le panache sédimentaire et calibrer les mesures (par capteurs optiques OBS) de MES ;

- péniche cible dont le positionnement-déplacement est instrumenté par GPS ;

- ligne de mesures pour l'obtention du courant, de la MES sur le fond et pour l'enregistrement des ondes de batillage à proximité de la berge.

$\mathrm{Au}$ total, 121 bateaux ont été enregistrés sur les deux sites durant les deux semaines de campagne de terrain : 24 ont été balisés en GPS, 70 étaient chargés. 


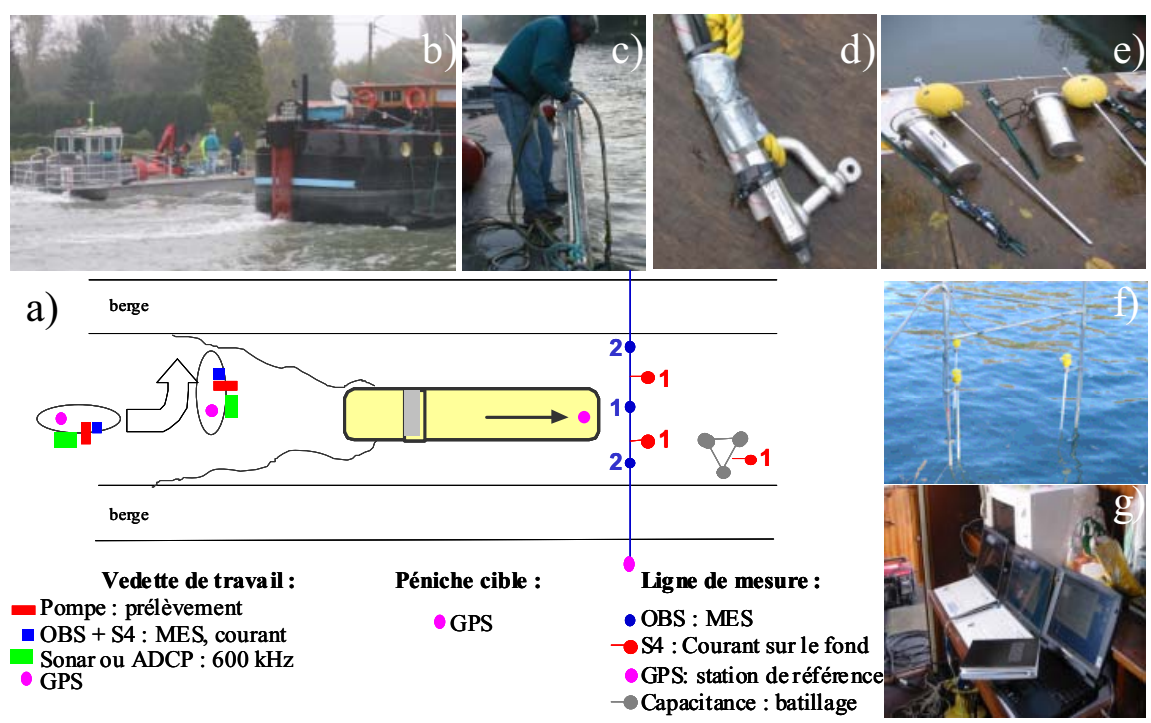

Figure 2 : Méthodologie et métrologie de la campagne de relevés : a) schéma de principe (vedette de travail, péniche cible, ligne de mesure) ; b) vedette de travail; c) prise d'échantillon ; d) prélèvement avec mesure $O B S$; e) OBS et $S 4$ de la ligne de mesure ; f) amplitude et direction du batillage ; g) acquisition.

\section{Transport des sédiments induits par les passages de bateaux}

\subsection{Re-suspension des sédiments}

Les données issues des capteurs OBS et $\mathrm{S} 4$ en provenance de la ligne de mesure (Fig. 3) fournissent une quantification du phénomène de re-suspension des sédiments. Obtenues à proximité du fond, les mesures mettent en évidence des pics de concentration et des pics de courant lors des passages des bateaux. Les enregistrements issus des GPS permettent d'identifier le bateau à l'origine des pics et d'en déterminer la vitesse au passage de la ligne de mesure. Enfin, la calibration des capteurs optiques OBS (concentration en MES) est réalisée à partir de prélèvements (vedette de travail) qui sont analysées par le Laboratoire Régionale de l'Est Parisien (LREP).
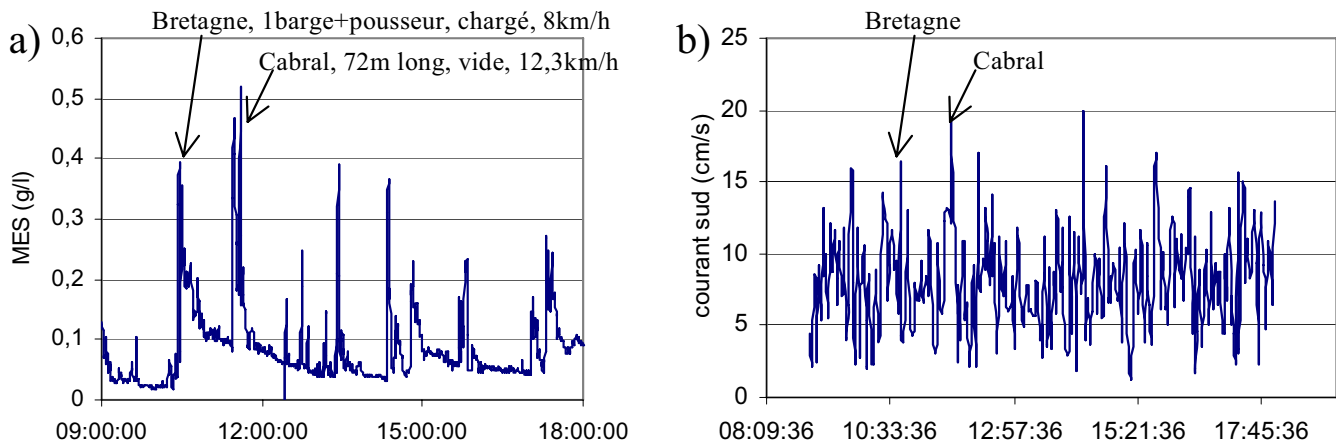

Figure 3 : Mesures sur le fond (site 1, 08/11/06) : a) pics de concentration (OBS sud, $\Delta t=30 \mathrm{~s})$; b) pics de courant ( $S 4 \mathrm{sud}, \Delta t=120 \mathrm{~s})$. 
Les résultats d'analyses des prélèvements d'eau (effectués à l'arrière des bateaux) et des mesures issues des capteurs OBS, mettent en évidence des valeurs aux pics de concentrations allant jusqu'à $712 \mathrm{mg} / \mathrm{l}$ pour le site 1 et $445 \mathrm{mg} / \mathrm{l}$ pour le site 2. Ces ordres de grandeur sont en accord avec les résultats disponibles sur le Mississipi (PARCHURE et al., 2001).

Les mesures de courant sur le fond ont été obtenues à basse fréquence d'acquisition $(\Delta \mathrm{t}=120 \mathrm{~s})$ et une estimation précise de la contrainte sur le fond à partir de l'énergie cinétique turbulente (par exemple VERNEY et al. 2007) n'a pas pu être réalisée. La relation classique, $\tau_{\mathrm{b}}=\rho \mathrm{u}^{* 2}$, a donc été considérée pour obtenir une estimation des contraintes de frottement : ces dernières varient entre 1 et $3 \mathrm{~Pa}$ pour le site 1 ; entre 5 et 11 Pa pour le site 2.

\subsection{Panache sédimentaire}

Les mesures de MES et de courant sur le fond permettent d'obtenir des informations sur l'érodabilité des fond au passage des bateaux. En revanche, elles ne permettent pas de décrire la formation, la persistance et le déclin d'un panache sédimentaire. Une solution est apportée par l'utilisation d'un ADCP placé sur la vedette de travail. Cette dernière se place derrière une péniche cible, et enregistre l'intensité du signal rétro-diffusé (reliée à la concentration en MES, d'après TESSIER et al. 2006) tout en maintenant une position fixe.

La figure 4 présente l'évolution spatiale et temporelle de l'intensité rétrodiffusée mesurée après le passage du bateau Aurelia (site 2, 100m de long, 9.6m de large, $1200 \mathrm{t}$ de chargement, progressant à $12 \mathrm{~km} / \mathrm{h}$ ). Elle met en évidence la succession de trois phases dans la dynamique du panache sédimentaire généré par le passage du bateau Aurelia :

- le profil à l'instant $\mathrm{t}=146 \mathrm{~s}$ correspond à un profil non perturbé par le bateau. Les profils suivants témoignent d'un flux de sédiments dirigée vers la surface libre: il s'agit du 'régime de re-suspension' mis en évidence au paragraphe précédent .

- Puis, à partir de $\mathrm{t}=191 \mathrm{~s}$, les profils se décalent vers le bas signifiant un 'régime de sédimentation partielle' jusqu'à $\mathrm{t}=260 \mathrm{~s}$.

- Enfin à partir de $\mathrm{t}=260 \mathrm{~s}$, une diminution des gradients verticaux de concentration est apparente, témoignant ainsi de la diffusion induite par le jet d'hélice : le 'régime de diffusion d'hélice' prend fin à $\mathrm{t}=415 \mathrm{~s}$.

Une comparaison des profils à $\mathrm{t}=146 \mathrm{~s}$ et à $\mathrm{t}=415 \mathrm{~s}$ suggère effectivement que l'effet du bateau Aurelia s'est entièrement dissipé et qu'une érosion nette, d'environ $10 \mathrm{~cm}$, s'est produite. Néanmoins, cette analyse suppose que la totalité du signal rétro-diffusé est liée aux MES : les effets des bulles d'air créées soit par le phénomène de cavitation à proximité des hélices soit par le déferlement des ondes de surface ne sont donc pas considérés. Plus précisément, leur présence est 
négligée en dessous de $92 \mathrm{~cm}$ de la surface libre, zone correspondante à une zone aveugle pour le capteur ADCP.

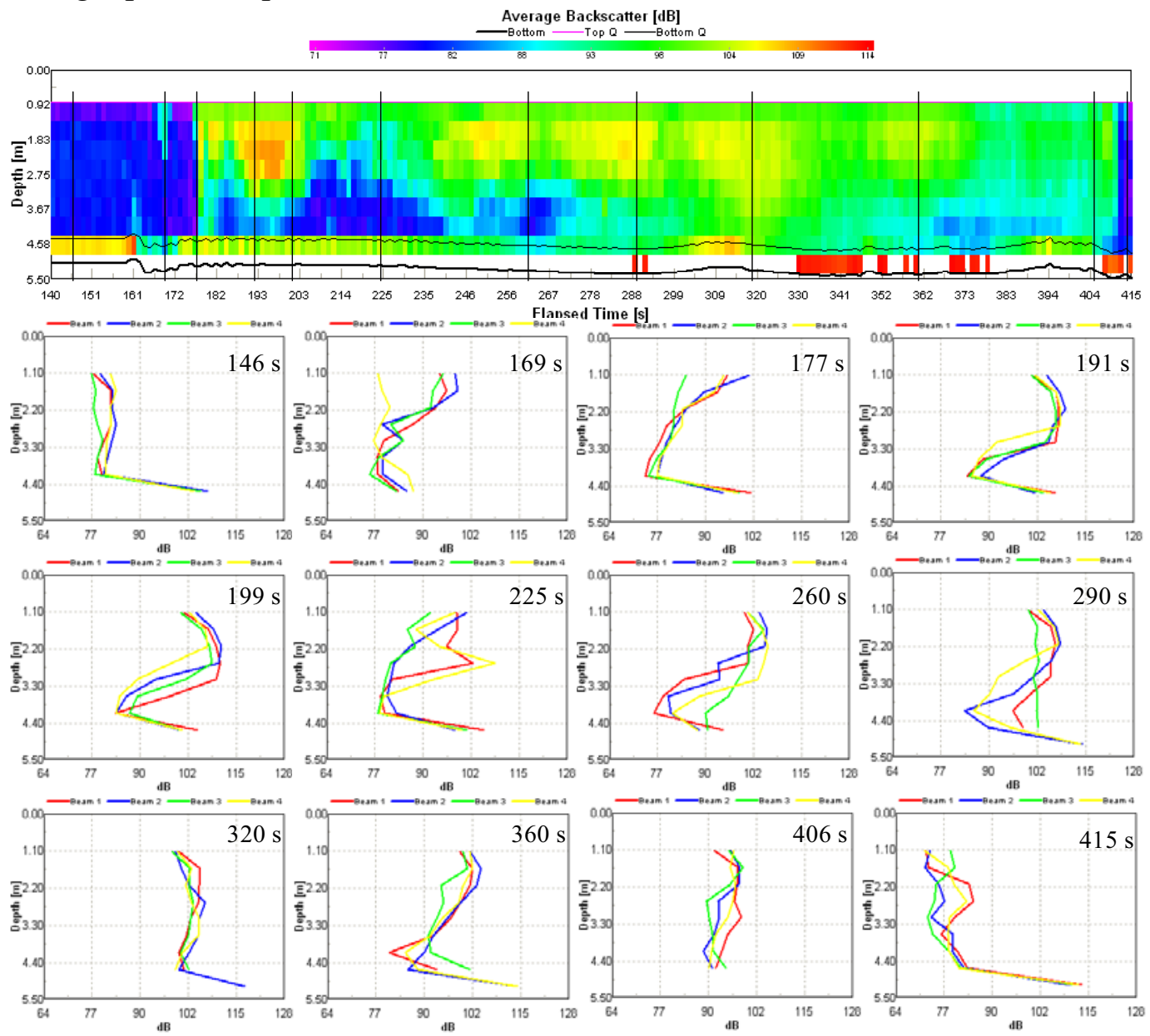

Figure 4 : Evolution temporelle (différentes distances derrière un bateau progressant à $12 \mathrm{~km} / \mathrm{h}$ ) des profils verticaux de l'intensité en $d B$ du signal rétrodiffusé (bateau Aurelia, 100m de long, 9.6m de large, $1200 t$ de chargement).

\section{$4 \quad$ Ondes de batillage observées sur la berge}

\subsection{Amplitude-fréquence des ondes}

Les hauteurs d'eau sont mesurées à partir d'un dispositif à trois jauges à capacitance placées au sommet d'un triangle équilatéral. Un exemple (site 1, convoi Tourisme, une barge et un pousseur, $652 \mathrm{t}$ de chargement, avançant à $8 \mathrm{~km} / \mathrm{h}$ ) est illustré sur la figure 5 .

Les différents phénomènes hydrodynamiques au passage d'un bateau sont illustrés par la figure 6 (issue du BAW, 2005). Une succession (onde de proue, abaissement du plan d'eau, onde divergente, onde transversale de poupe, onde secondaire) est observée en un point fixe de la berge. 

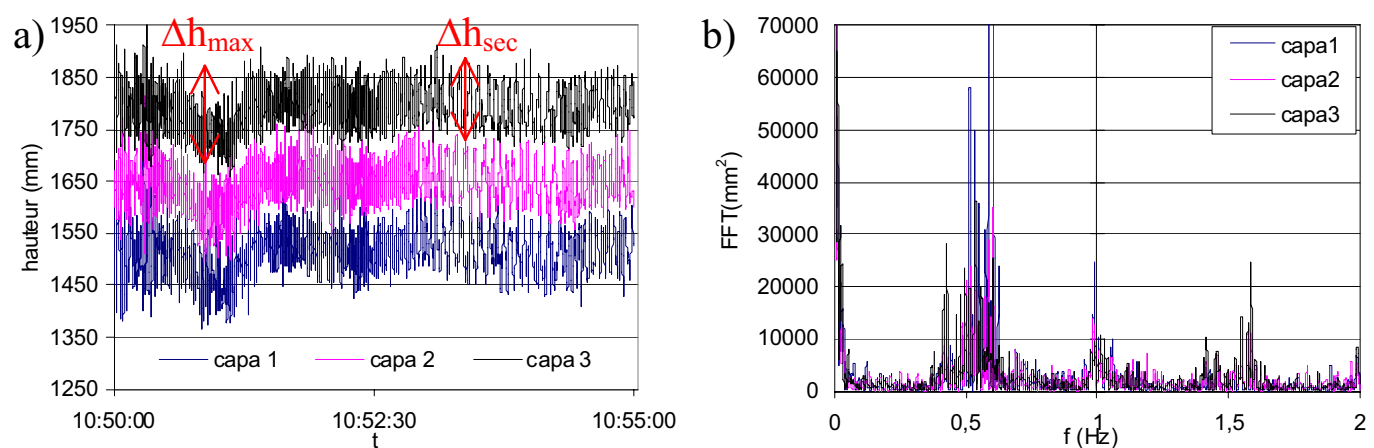

Figure 5 : Mesures issues du dispositif de batillage à trois points fixés sur la berge : a) évolution de la hauteur d'eau ; b) transformée de Fourier.
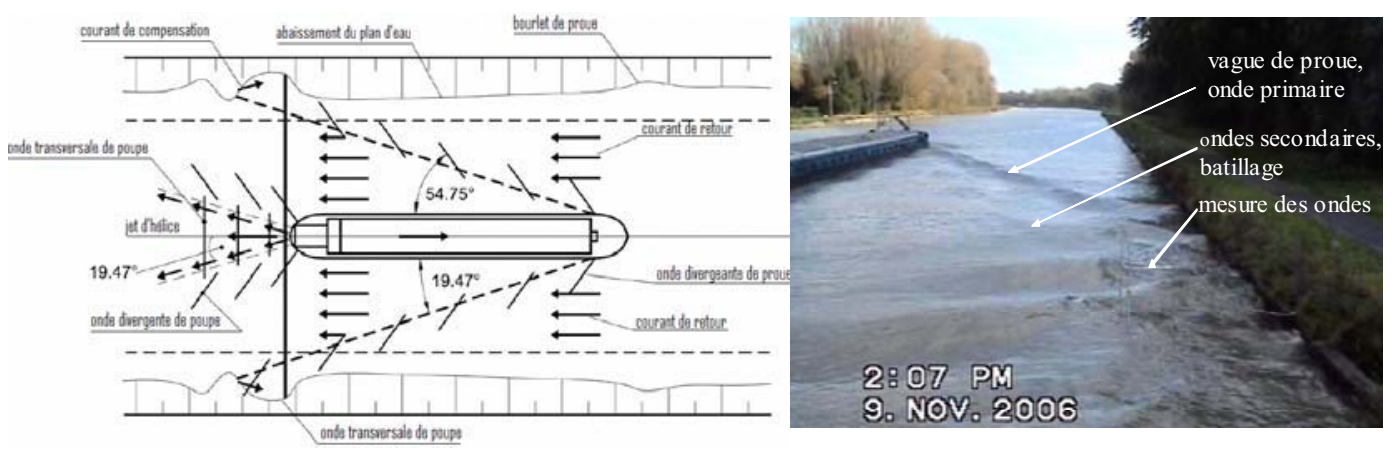

Figure 6: Champ de vagues induit par le passage d'un bateau dans une voie d'eau confinée: a) définitions (BAW, 2005) ; b) passage du bateau Dhana (site 1, $38 m$ de long).

Les différents phénomènes, en particulier l'onde primaire (de proue) et les ondes secondaires, ont des amplitudes (Fig. 5a) et des fréquences (Fig. 5b) différentes : une localisation temporelle de l'occurrence d'une fréquence est impossible par transformation de Fourier; elle est rendue possible à partir d'une analyse aux ondelettes (\$4.2). Selon le type des ondes (de poupe ou secondaire) considérées pour la formulation empirique des relations 'hauteur de vague-vitesse d'avance du bateau', une différence est observée. Une comparaison entre les mesures et les prédictions obtenues à partir de deux formules empiriques a été menée (Fig. 7) : la formule du BAW, 2005 (Eq. 1) et celle du Port Autonome du Havre, 1979 (Eq. 2) :

$$
\begin{aligned}
& \Delta \mathrm{h}_{\text {sec }}=0.5 \frac{\mathrm{V}^{8 / 3}}{\mathrm{~g}^{4 / 3} \mathrm{D}^{1 / 3}} \\
& \Delta \mathrm{h}_{\text {max }}=0.07 \frac{\mathrm{V}^{2}}{\mathrm{D}^{1 / 3}}
\end{aligned}
$$

avec $\Delta \mathrm{h}_{\max }\left(\Delta \mathrm{h}_{\text {sec }}\right)$ l'amplitude maximale (amplitude des ondes secondaires) définies sur la figure $5 \mathrm{a}, \mathrm{V}$ la vitesse du bateau $(\mathrm{m} / \mathrm{s})$ et $\mathrm{D}$ la distance entre le bateau et le point de mesure (m). 

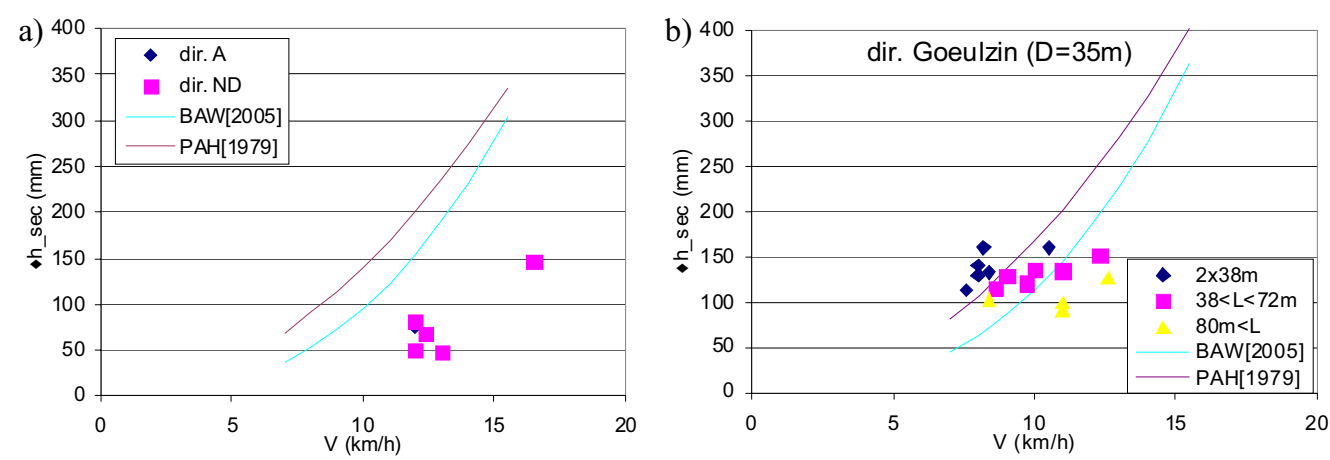

Figure 7 : Comparaison entre l'amplitude des ondes primaires et secondaires mesurés in situ et les formules empiriques (Eq. 1) et (Eq. 2).

Les résultats présentés sur la figure 7 mettent en évidence un bon accord entre mesures et prédictions pour les amplitudes des ondes secondaires relevées sur le site 1 mais un désaccord pour les mesures sur le site 2 .

\subsection{Direction des ondes et analyse fréquence-temps}

Le dispositif de mesure des ondes à trois points fixes permet en outre de déterminer les déphasages entre les mesures, et par conséquent, donne accès à la direction des ondes. A partir d'une fonction de corrélation croisée (Eq. 3), ces déphasages sont déterminées automatiquement : les résultats de cette étude met en évidence que la direction des ondes impactant la berge est orientée à $54^{\circ}\left( \pm 6^{\circ}\right)$ par rapport à l'axe de navigation. Cette direction correspond à celles des ondes divergentes (Fig. 6).

$\mathrm{XCF}_{\mathrm{fg}}(\tau)=\int_{\mathrm{t}=0}^{\infty} \mathrm{f}(\mathrm{t}) \mathrm{g}(\mathrm{t}+\tau) \mathrm{dt}$

Par ailleurs, la transformation des signaux de batillage en ondelettes (fonctions oscillantes bien localisées en temps et en fréquence) permet de visualiser les signaux dans l'espace fréquence-temps et de déterminer l'occurrence (naissance, persistance et déclin) d'un mode fréquentiel. La figure 8 présente les résultats d'une analyse aux ondelettes pour le cas présenté en figure 5 .

La visualisation du signal issue des jauges de hauteurs d'eau dans la fenêtre fréquence - temps fournit des informations précieuses sur le phénomène : non seulement l'information sur les fréquences est identique à un traitement par analyse de Fourier (les modes à $0,5,1$ et $1,5 \mathrm{~Hz}$ de la figure $5 \mathrm{~b}$ sont retrouvées), mais aussi l'instant à laquelle les hautes fréquences (entre 1.4 et $1.7 \mathrm{~Hz}$ ) se produisent.

La non simultanéité des fréquences (CAMPION, 1999) est confirmée dans cette fenêtre et la transition de régimes 'ondes primaires - ondes secondaires' est précisément localisée. 


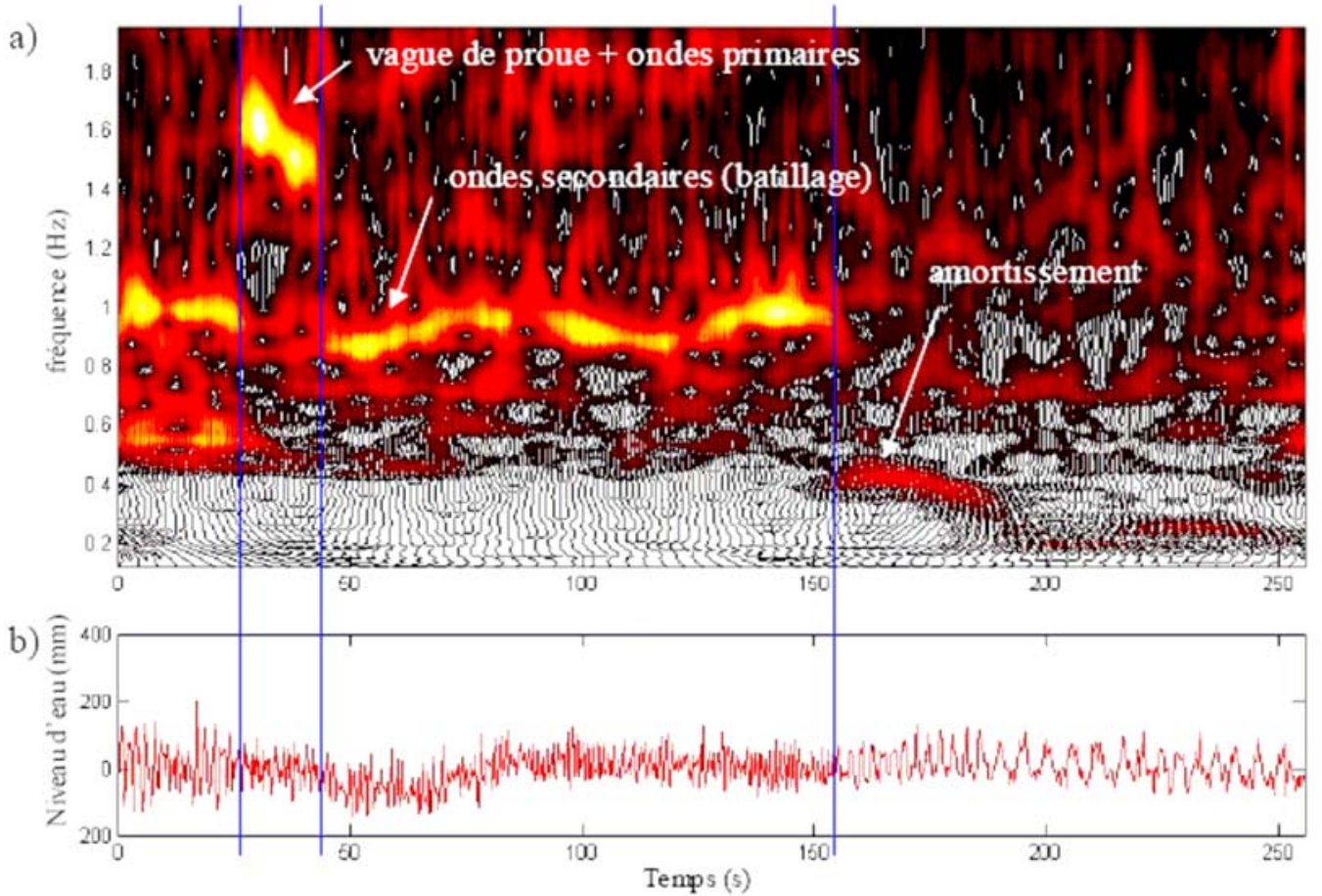

Figure 8: Analyse aux ondelettes (cas présenté en figure 5) : a) isocontours de l'énergie dans la fenêtre fréquence-temps ; b) signal du capteur capal.

\section{$5 \quad$ Conclusions}

La caractérisation et la quantification de l'impact de la navigation sur le transport des sédiments présentent un intérêt double, d'ordre écologique (transfert de contaminant) et d'ordre économique pour l'entretien de la voie d'eau.

Dans cette étude, elles sont obtenues par des relevés de terrain portant sur la remise en suspension des sédiments et le batillage. L'analyse du panache sédimentaire à partir du signal ADCP rétro-diffusé a permis d'identifier trois régimes: le premier correspond à une érosion du fond, le second à une sédimentation partielle, le dernier à une diffusion sous l'effet des hélices. Trois modes fréquentiels ont également été mis en évidence par une transformation classique de Fourier: une transformation aux ondelettes est pertinente pour démontrer que l'occurrence de ces modes n'est pas simultanée.

Des efforts de recherche sont nécessaires pour compléter cette étude et formuler des recommandations dans le cas des berges naturelles ou végétalisées.

\section{$6 \quad$ Remerciements}

L'étude a été co-financée par les Voies Navigables de France (VNF) et par le Ministère de l'Ecologie, de l'Energie, du Développement Durable et de l'Aménagement du Territoire (MEEDDAT). Les auteurs remercient les services de 
l'Etat qui sont intervenus pour la réalisation du programme de travail (l'équipe de plongeurs du Laboratoire Régional de l'Est Parisien, LREP, ainsi que les pilotes des Services de la Navigation du Nord Pas-de-Calais, SNNPC, et de la Seine, SNS). Le Conservatoire National des Arts et Métiers Haute-Normandie (CNAM Haute Normandie) est également remercié pour le prêt d'une péniche. La participation de Prof. Brian Morse (Université Laval, Québec) à la définition du protocole expérimental, la mise à disposition du capteur ADCP par Dr. Sottolichio (U. Bordeaux, Laboratoire EPOC) et du code aux ondelettes par Dr Coutel (INRETS) ont été vivement appréciés.

\section{$7 \quad$ Références bibliographiques}

1 BAW (2005): Principles for the Design of Bank and Bottom Protection for Inland Waterways, bulletin $\mathrm{n}^{\circ} 88$ of Bundesanstalt für Wasserbau, $125 \mathrm{p}$.

2 CAMPION V. (1999) : Le batillage sur les berges de Saône, Travail de fin d'étude de l'ENTPE, $54 \mathrm{p}$.

3 GARCIA M, ADMIRAAL D., RODRIGUEZ J. (1999): Laboratory experiments on navigation-induced bed shear stresses and sediment resuspension, Int. Jour. Sed. Res. 14(2), pp 303-317.

4 KOSLOWSKI J. (2007) : Ship-induced riverbank and harbour damage Evidence for claims processing, Hydro International (sept. 2007), pp. 2-4.

5 MOULIN B., JABEUR N., MORSE B. (2003): Benefits of a multi-agent approach to solve the problem of ship course planning on the St Lawrence River, Journal Geomatica, Vol 56, pp 35-43.

6 PARCHURE T.M., MCANALLY W.H., TEETER A.M. (2001) : Wave-Induced Sediment Resuspension near the Shorelines of the Upper Mississippi River System, (ENV Report 20), 99p

7 ROCHETTE P. (2008): Techniques de protection des berges en génie végétal, spécificités des voies navigables, notes manuscrites du colloque VNF-CETMEF 'Protection des berges', Nancy, 2-3 avril 2008.

8 TESSIER C. (2006): Caractérisation et dynamique des turbidités en zone côtière : l'exemple de la région maritime Bretagne Sud, Thèse, 385 p.

9 VERNEY R., DELOFFRE J., BRUN-COTTAN J.C., LAFITE R. (2007) : The effect of wave-induced turbulence on intertidal mudflats : impact of boat traffic and wind, Continental Shelf Research, Vol. 27(5), 594-612.

10 WOLTER C. AND ARLINGHAUS R. (2003) : Navigation impacts on freshwater fish assemblages : the ecological relevance of swimming performance, Reviews in Fish Biology and Fisheries 13, pp 63-89. 